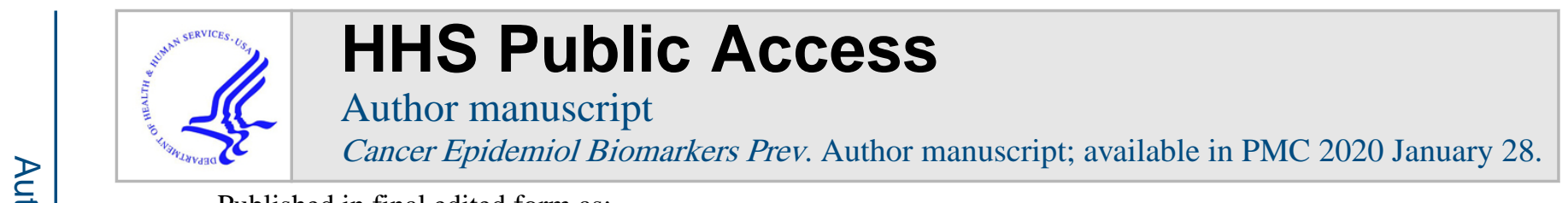

Published in final edited form as:

Cancer Epidemiol Biomarkers Prev. 2017 December ; 26(12): 1730-1735. doi:

10.1158/1055-9965.EPI-17-0495.

\title{
Metabolic Obesity Phenotypes and Risk of Breast Cancer in Postmenopausal Women
}

\author{
Geoffrey C. Kabat ${ }^{1}$, Mimi Y. Kim ${ }^{1}$, Jennifer S. Lee ${ }^{2,3}$, Gloria Y. Ho ${ }^{4}$, Scott B. Going ${ }^{5}$, \\ Jennifer Beebe-Dimmer ${ }^{6}$, JoAnn E. Manson ${ }^{7}$, Rowan T. Chlebowski ${ }^{8}$, Thomas E. Rohan ${ }^{1}$ \\ ${ }^{1}$ Department of Epidemiology and Population Health, Albert Einstein College of Medicine, Bronx, \\ New York \\ ${ }^{2}$ Departments of Medicine and Health Research and Policy, Stanford University Medical Center, \\ Stanford, California \\ ${ }^{3}$ Palo Alto VA Health Care System, Stanford, California \\ ${ }^{4}$ Department of Occupational Medicine, Epidemiology and Prevention, Feinstein Institute for \\ Medical Research, Hofstra Northwell School of Medicine, Great Neck, New York \\ ${ }^{5}$ Department of Nutrition Sciences, University of Arizona, Tucson, Arizona \\ ${ }^{6}$ Department of Oncology, Wayne State University School of Medicine, Detroit, Michigan \\ ${ }^{7}$ Division of Preventive Medicine, Brigham and Women's Hospital, Harvard Medical School, \\ Boston, Massachusetts \\ ${ }^{8}$ City of Hope National Medical Center, Duarte, California
}

\begin{abstract}
Background: Obesity and the metabolic syndrome (MetS) have both been linked to increased risk of postmenopausal breast cancer; however, their relative contributions are poorly understood.

Methods: We examined the association of metabolic phenotypes of obesity defined by presence of the MetS (yes and no) and body mass index (BMI; normal, overweight, obese) with risk of
\end{abstract}

\footnotetext{
Reprints and Subscriptions To order reprints of this article or to subscribe to the journal, contact the AACR Publications Department at pubs@ aacr.org.Permissions To request permission to re-use all or part of this article, use this link http://cebp.aacrjournals.org/ content/26/12/1730.

Corresponding Author: Geoffrey C. Kabat, Albert Einstein College of Medicine, 1300 Morris Park Avenue, Belfer 1301, Bronx, NY 10461. Phone: 718-430-3038; Fax: 718-430-8653; geoffrey.kabat@einstein.yu.edu.

Authors' Contributions

Conception and design: G.C. Kabat, J.E. Manson, R.T. Chlebowski, T.E. Rohan

Development of methodology: G.C. Kabat, T.E. Rohan

Acquisition of data (provided animals, acquired and managed patients, provided facilities, etc.): G.C. Kabat, J.E. Manson, R.T. Chlebowski, T.E. Rohan

Analysis and interpretation of data (e.g., statistical analysis, biostatistics, computational analysis): G.C. Kabat, M.Y. Kim, J.S. Lee, G.Y. Ho, R.T. Chlebowski, T.E. Rohan

Writing, review, and/or revision of the manuscript: G.C. Kabat, M.Y. Kim, J.S. Lee, G.Y. Ho, S.B. Going, J. Beebe-Dimmer, J.E. Manson, R.T. Chlebowski, T.E. Rohan

Administrative, technical, or material support (i.e., reporting or organizing data, constructing databases): G.C. Kabat, J.E.

Manson, T.E. Rohan

Study supervision: G.C. Kabat, T.E. Rohan

Disclosure of Potential Conflicts of Interest

No potential conflicts of interest were disclosed.
} 
postmenopausal breast cancer in a prospective analysis of a cohort of postmenopausal women ( $n$ $21,000)$ with baseline measurements of blood glucose, triglycerides, HDL-cholesterol, blood pressure, waist circumference, and BMI. Women were classified into 6 metabolic obesity phenotypes according to their BMI $\left(18.5-<25.0,25.0-<30.0, \geq 30.0 \mathrm{~kg} / \mathrm{m}^{2}\right)$ and presence of the MetS ( $\geq 3$ of the following: waist circumference $\geq 88 \mathrm{~cm}$, triglycerides $\geq 150 \mathrm{mg} / \mathrm{dL}$, HDL-C $<50$ $\mathrm{mg} / \mathrm{dL}$, glucose $\geq 100 \mathrm{mg} / \mathrm{dL}$, and systolic/diastolic blood pressure $\geq 130 / 85 \mathrm{mmHg}$ or treatment for hypertension). HRs for incident breast cancer and 95\% confidence intervals (95\% CI) were estimated using Cox proportional hazards models.

Results: Over 15 years of follow-up, 1,176 cases of invasive breast cancer were diagnosed. Obesity, regardless of metabolic health, was associated with increased risk of breast cancer. Being obese and metabolically unhealthy was associated with the highest risk: HR, 1.62; 95\% CI, 1.331.96. These associations were stronger in women who had never used hormone therapy.

Conclusions: Our findings suggest that both obesity and metabolic dysregulation are associated with breast cancer risk.

Impact: Beyond BMI, metabolic health should be considered a clinically relevant and modifiable risk factor for breast cancer.

\section{Introduction}

The prevalence of obesity has been increasing in the United States over the past three decades (1). At present, roughly one-third of U.S. adults are overweight [body mass index (BMI) of $25.0-<30.0 \mathrm{~kg} / \mathrm{m}^{2}$ ], and one-third are obese (BMI $\geq 30 \mathrm{~kg} / \mathrm{m}^{2}$; ref. 1). In addition, there has been an increase in the prevalence of diabetes and of the metabolic syndrome (MetS; 2-4). The utility of the MetS is subject to controversy (5), and definitions of the syndrome vary widely and, depending on the definition used and the population studied, the prevalence of the MetS ranges from $10 \%$ to $34 \%$ (6). Both obesity and the MetS are associated with increased risks of cardiovascular disease, diabetes, and mortality, but there is disagreement as to their relative contributions (7-10).

Obesity is an established risk factor for postmenopausal breast cancer (11), and, in addition, there is limited evidence that both the MetS and diabetes are associated with increased risk of the disease (12-15). However, to date, only three studies have examined the relative contributions of BMI, or waist circumference, and the MetS (or insulin resistance syndrome) to breast cancer risk (16-18). Two of these had relatively small sample size, and all three dichotomized BMI into normal weight and overweight/obese.

To address this question, considering the full range of BMI, we examined the contributions of BMI and the MetS to risk of breast cancer in a subset of nearly 21,000 participants in the Women's Health Initiative cohort with measurements of BMI and components of the MetS.

\section{Materials and Methods}

The Women's Health Initiative (WHI) is a large, multicenter study designed to improve our understanding of the determinants of major chronic diseases in postmenopausal women. It is composed of a clinical trial component $(\mathrm{CT}, n=68,132)$ and an observational study 
component (OS, $n=93,676$; ref. 19). The CT component included four randomized controlled intervention studies: hormone therapy (two trials), low-fat dietary modification, and calcium + vitamin D supplementation. Women between the ages of 50 and 79 and representing the major racial/ethnic groups were recruited from the general population at 40 clinical centers throughout the United States between 1993 and 1998. Details of the study design and reliability of the baseline measures have been published $(19,20)$. Written informed consent was obtained from participants at all WHI centers in accordance with recognized ethical guidelines, and the study was approved by the institutional review board of each center, as well as by that of the Coordinating Center at the Fred Hutchinson Cancer Research Center.

At study entry, self-administered questionnaires were used to collect information on demographics, medical, reproductive, family history of cancer, and lifestyle factors, including smoking history, alcohol consumption, dietary habits, and recreational physical activity. All participants had their weight and height measured by trained staff at baseline. Weight was measured to the nearest $0.1 \mathrm{~kg}$, and height to the nearest $0.1 \mathrm{~cm}$. BMI was computed as weight in kilograms divided by the square of height in meters. Two blood pressure measurements were obtained $\geq 30$ seconds apart, and the average of the 2 measurements was used in the analysis. Questions about physical activity at baseline referred to a woman's usual pattern of activity, including walking and recreational physical activity. A variable "current total leisure-time physical activity" (MET-h/wk) was computed by multiplying the number of hours per week of leisure-time physical activity by the metabolic equivalent (MET) value of the activity and summing over all types of activities (21).

\section{Follow-up and ascertainment of outcomes}

Clinical outcomes (including new cancer diagnoses) were updated semiannually in the CT and annually in the OS using in-person, mailed, or telephone questionnaires. Self-reports of malignancy were verified by central review of medical records and pathology reports by trained physician adjudicators (22).

\section{The CVD biomarkers subsample}

Individuals who had baseline measurements of fasting serum glucose and insulin and other clinical parameters that were made in various substudies within WHI were assembled into the CVD Biomarkers Subsample ( $n=25,446)$. Some substudies entailed selecting a random sample; others selected participants based on specific age and ethnicity/race criteria within the hormone therapy trials; another substudy was a nested case-control study within the hormone therapy trials with random sampling of controls.

\section{Assays for glucose, HDL-C, and triglycerides}

Blood was obtained after at least 8 hours of fasting for $99.8 \%$ of participants in the Subsample. The specimens were centrifuged, and serum and plasma were frozen at $-70^{\circ} \mathrm{C}$ and shipped on dry ice to a central processing facility. HDL-C was measured in serum using the HDLC Plus 3rd Generation Direct Method (Roche) on the Roche Modular P Chemistry Analyzer. Triglycerides were measured in serum using Triglyceride GB reagent (Roche) on 
the Roche Modular P Chemistry Analyzer. In the vast majority of women $(n=22,314,88 \%)$, glucose was measured in serum using the Gluco-quant Glucose/hexokinase reagent (Roche Diagnostics) on the Roche Modular P Chemistry analyzer (Roche Diagnostics Corporation). In the remainder of the women, serum glucose was determined by the hexokinase method on the Hitachi 747 (Boehringer Mannheim Diagnostics).

\section{Definition of the MetS}

We used the National Cholesterol Education Program (NCEP) Adult Treatment Panel III (ATP III) definition of the MetS (4): having $\geq 3$ of the 5 following criteria: waist circumference $>88 \mathrm{~cm}$, triglycerides $\geq 150 \mathrm{mg} / \mathrm{dL}$, HDL-C $<50 \mathrm{mg} / \mathrm{dL}$, glucose $\geq 100 \mathrm{mg} / \mathrm{dL}$, and systolic/diastolic blood pressure $\geq 130 / 85 \mathrm{mmHg}$ or treatment for hypertension. In addition to the MetS, we computed homeostasis model assessment-insulin resistance (HOMA-IR), a measure of insulin resistance, using the formula [fasting glucose $(\mathrm{mg} / \mathrm{dL}) \times$ fasting insulin $(\mathrm{mg} / \mathrm{dL})] / 405$ (23), which was used in the study by Gunter and colleagues (17) as an index of metabolic health.

\section{Exclusions}

For the purposes of the present analysis, baseline BMI and components of the MetS were available for 23,900 (99\%) of 24,210 women in the subsample. We excluded women with diabetes reported at baseline $(n=2,440)$, women missing waist circumference measurements $(n=53)$, and women with a history of breast cancer $(n=557)$. After exclusions, 20,944 women were available for analysis (88\% of women with information on MetS and BMI), among whom, as of September 20, 2015, 1,176 incident invasive breast cancer cases had occurred.

\section{Statistical analysis}

Cox proportional hazards models were used to estimate hazard ratios (HR) and 95\% confidence intervals (CI) for the associations of interest. The outcome was time to diagnosis of breast cancer. Participants who had not developed breast cancer by the end of follow-up, who had died, or who withdrew from the study before the end of follow-up were censored. Cases contributed persontime to the study from their date of enrollment until the date of diagnosis, and noncases (participants who were censored) contributed person-time from their date of enrollment until the end of follow-up (September 20, 2015), date of death, or date of withdrawal from the study, whichever came first. We examined both age-adjusted and fully adjusted models, which included breast cancer risk factors as well as other potential confounding variables. Because the results differed little, we present the fully adjusted results.

We examined the association of categories of BMI (18.5-<25.0 - normal weight, 25.0$<30.0$ - overweight, and $230.0 \mathrm{~kg} / \mathrm{m}^{2}$ - obese) and of presence of the MetS, separately and with mutual adjustment, with risk of breast cancer. We then estimated risk after crossclassifying women by categories of BMI and presence of the MetS simultaneously, yielding six groups: metabolically healthy/normal weight (MHNW); metabolically unhealthy/normal weight (MUNW); metabolically healthy/overweight (MHOW); metabolically unhealthy/ 
overweight (MUOW); metabolically healthy/obese (MHO); and metabolically unhealthy/ obese (MUO).

Because the MetS is a composite of different clinical factors, we further examined the association of each component with breast cancer risk. In addition, we examined the association of combinations of metabolic health defined by HOMA-IR ("low" = lowest quartile/"high" = quartiles 3 and 4) and BMI (normal, overweight, obese) with breast cancer risk.

Tests for linear trend were performed by assigning the median value to each category and modeling the variable as a continuous variable.

We conducted two sensitivity analyses: we excluded the first 3 years of follow-up to address the possibility of reverse causation (effects of subclinical cancer on body weight and metabolic status); also, because waist circumference is strongly correlated with BMI, we repeated the analyses after excluding waist circumference from the definition of the MetS and defining presence of the syndrome as $\geq 2$ of the four remaining components.

We tested the proportional hazards assumption using PROC LIFETEST (SAS Institute). None of the formal tests for nonproportional hazards and the log-log survival plots indicated any marked deviation from nonproportionality.

All analyses were performed in SAS 9.4 (SAS Institute). All $P$ values are two-sided.

\section{Results}

The metabolic obesity phenotypes showed differences by demographic and behavioral characteristics (Table 1). Within BMI categories, compared with metabolically healthy women, metabolically unhealthy women tended to be older and to have fewer years of education, and were more likely to be white. Both alcohol intake and MET-h/wk of physical activity showed decreasing trends from MHNW to MUO; frequency of mammographic screening (ever and in the past 5 years) varied little across obesity phenotypes. As expected, serum glucose, triglycerides, HDL-C, and hypertension differed mainly by presence of the MetS (higher in those with the MetS), whereas serum insulin and waist circumference were positively associated both with BMI and with poor metabolic status.

Both BMI and the MetS, considered individually, were positively associated with breast cancer: HRs for overweight and obese women were 1.12 (95\% CI, 0.95-1.33) and 1.51 (95\% CI, 1.28-1.78), p for trend <0.0001, respectively; women with the MetS had a HR of 1.28 (95\% CI, 1.13-1.44; Table 2). After mutual adjustment, the HRs for BMI and metabolic status were somewhat attenuated; however, the HR for obese women and the trend with BMI as well as the HR for the MetS remained statistically significant. A similar pattern was seen when MetS and tertiles of waist circumference were considered separately and together in the same model.

The association of metabolic obesity phenotypes with breast cancer is presented in Table 3 . Relative to women who were MHNW, risk of breast cancer was unchanged in women who 
were MUNW or MHOW; however, the number of MUNW women was small. Metabolically unhealthy overweight women had a nonsignificantly increased risk.

Women who were MHO and MUO were at increased risk, with the highest risk evident among MUO women: HR 1.61 (95\% CI, 1.34-1.94). When the analysis was restricted to never users of hormone therapy and to women who were not in the treatment arms of the hormone therapy clinical trials, the associations with MHO and MUO were strengthened. In addition, when the first 3 years of follow-up were excluded to address possible reverse causality, the associations were slightly strengthened compared with those for all subjects.

In the sensitivity analysis in which waist circumference was excluded from the definition of the MetS [due to its strong correlation with BMI $(r=0.8)$ ], the HRs for metabolic obesity phenotypes relative to metabolically healthy normal weight women were virtually unchanged.

We examined the association of each individual component of the MetS with breast cancer, with all covariates including BMI in the model. Blood glucose, triglycerides, HDL-C were not associated with breast cancer (HR for highest vs. lowest quartile: 1.07 (95\% CI, 0.901.29), p for trend $0.43 ; 1.16$ (95\% CI, 0.97-1.39), $P_{\text {trend }} 0.09$, and 0.88 (95\% CI, 0.74-1.05, $\mathrm{p}$ for trend 0.10, respectively). Hypertension was also not associated with risk: HR for presence versus absence of hypertension at baseline 1.02, 95\% CI, 0.89-1.16. Waist circumference showed a positive association with breast cancer risk when BMI was not included in the model: HR for middle and upper tertiles: 1.20 (95\% CI, 1.03-1.39) and 1.47 (95\% CI, 1.27-1.72), $P_{\text {trend }}<0.0001$.

Combinations of HOMA-IR (lowest quartile vs. 2 highest quartiles) and BMI (18.5-<25.0, $25.0-<30.0,230.0 \mathrm{~kg} / \mathrm{m}^{2}$ ) were examined to isolate insulin resistance instead of the MetS (Table 4). Only women who were obese and had high HOMA-IR had significantly elevated risk of breast cancer. The HR for obese women with low HOMA-IR was elevated but not statistically significant.

\section{Discussion}

In the present study, both obesity and being metabolically unhealthy were each individually associated with increased risk of breast cancer. After mutual adjustment, both associations remained significant. Relative to MHNW women, only MHO and MUO women were at increased risk, whereas HRs for MUNW, MHOW, and MUOW were close to unity. Exclusion of waist circumference from the definition of metabolic health did not alter the associations. When women were cross-classified by HOMA-IR and BMI, only women who were both obese and had high HOMA-IR were at increased risk; however, the number of cases in some subgroups was small.

The three previous studies that have examined metabolic obesity phenotypes in relation to breast cancer all used different markers of metabolic status: glucose levels (16), HOMA-IR (17), and an approximation of the MetS, based on central obesity, elevated blood pressure, type 2 diabetes, and dyslipidemia (18). All three studies contrasted overweight and obese women combined to normal weight women. Using data from the Framingham Heart Study, 
Moore and colleagues (16) found that overweight/obese women with elevated glucose levels had a 2.6-fold increased risk (95\% CI, 1.4-4.9) of female reproductive cancers and postmenopausal breast cancer $(n=171)$, whereas normal weight women with elevated glucose had no excess risk. Overweight/obese women with normal glucose levels had a 1.7fold (95\% CI, 1.1-2.5) increased risk.

In an analysis of a case-cohort study within the Women's Health Initiative, Gunter and colleagues (17) reported that metabolically healthy overweight/obese women, with metabolic health defined using HOMA-IR, were not at elevated risk of breast cancer compared with metabolically healthy normal weight women (HR 0.96, 95\% CI, 0.64-1.42), whereas women with elevated HOMA-IR (>median) had elevated risk when they were either overweight/obese or normal weight, compared to normal weight women with normal HOMA-IR; however, the latter HR was not significant (HRs 1.76, 95\% CI, 1.19-2.60 and $1.80,95 \%$ CI, $0.88-3.70$, respectively). The authors concluded that metabolic health may be more biologically relevant and more useful for breast cancer risk stratification than adiposity per se.

Using data from the Sister Study, Park and colleagues (18) observed that, compared with normal weight women with no metabolic abnormalities, normal weight women with $\geq 1$ metabolic abnormality and overweight/obese women with no metabolic abnormalities had similarly increased risk of postmenopausal breast cancer: HR, 1.26; 95\% CI, 1.01-1.56 and 1.24; 95\% CI, 0.99-1.55, respectively. Park and colleagues (18) did not have blood glucose, insulin, or lipid measurements, and used a diagnosis of diabetes or use of diabetes medication and cholesterol-lowering medications to assess metabolic health. In addition, the authors defined metabolic health as having none of 4 factors, whereas most definitions of metabolic health allow for 1 or 2 factors.

Our results underscore the importance of examining the full range of BMI when investigating the relative contributions of obesity and metabolic dysregulation to the risk of breast cancer. Combining overweight and obese subjects, as has been done in previous studies, dilutes the trend toward increasing risk with increasing levels of BMI in combination with metabolic status. Our findings suggest that both increasing adiposity (whether measured by BMI or waist circumference) and metabolic status make independent and joint contributions to the risk of breast cancer. Both presence of the MetS and its individual components (other than waist circumference) showed weak or nonsignificant associations with breast cancer. In contrast, HOMA-IR was significantly associated with risk, and the association of the MetS with breast cancer appears to reflect this, because HOMA-IR is robustly associated with the MetS (41\% of women with the MetS were in the highest quartile of HOMA-IR compared with $7.5 \%$ of women without the MetS ( $P<$ 0.0001 ). Our analysis indicated that waist circumference did not did not show a stronger association with breast cancer compared to BMI and that removing waist circumference from the definition of the MetS did not affect the association of metabolic obesity phenotypes with breast cancer.

Consistent with the analysis of Gunter and colleagues (17), in our analysis, obese women with high HOMA-IR were at increased risk of breast cancer. Normal weight women with 
high HOMA-IR were not at increased risk in our study, and the HR for normal-weight women with high HOMA-IR was not statistically significant in Gunter and colleagues. The numbers of cases among overweight women with either high or low HOMA-IR in our study were small. The total number of cases in our analysis was 1,176 compared with 497 in Gunter and colleagues (17), permitting us to subdivide the $\geq 25 \mathrm{~kg} / \mathrm{m}^{2}$ group into $25.0-<30$ and $330.0 \mathrm{~kg} / \mathrm{m}^{2}$. Overlap between the present study population and that of Gunter and colleagues (17) is minimal (on the order of 20\%). It should be noted that in both analyses the numbers of cases among women with normal weight who were metabolically unhealthy (Gunter and colleagues; ref. 17), and among women with the MUNW and MUOW phenotypes in the present study were small, so that it is not possible to rule out an elevated risk of breast cancer in these groups. The findings from the present analysis regarding the associations of BMI and HOMA-IR with breast cancer are consistent with those concerning the association of insulin and glucose with risk of breast cancer in this same cohort (24).

Obesity and the MetS are overlapping in their biological pathways influencing risk of postmenopausal breast cancer. Insulin resistance can be a consequence of obesity but can have genetic determinants as well (4). Increased estrogen levels resulting from the aromatization of androgens in adipose tissue may promote cell proliferation in breast tissue (25). Second, elevated insulin concentrations resulting from insulin resistance may exert promitotic and anti-apoptotic effects $(26,27)$ and stimulate cell-cycle progression in breast cancer cells (28). Prolonged hyperinsulinemia may additionally result in increased levels of free or bioactive IGF-1, which also promotes signaling pathways favoring tumor development (25). Finally, inflammatory cytokines, including tumor necrosis factor-a, interleukin 6 , and prostaglandin E2, may contribute to breast carcinogenesis by promoting cell proliferation and cell-cycle progression $(25,29,30)$. These molecular factors interfere with cell signaling in the PI3K-AKT-mTOR pathway, which regulates cell-cycle progression, apoptosis, and protein synthesis (29-31).

Strengths of the present study include its large sample size, the central adjudication of all breast cancer diagnoses, the availability of fasting blood samples on all participants, and measured anthropometric variables, including all components of the MetS. Limitations include: small numbers of cases in some subgroups defined by BMI and metabolic health, the lack of information on change in metabolic phenotype and adiposity over time, and the lack of assessment of medications, including statins and metformin. Although we excluded women with prevalent diabetes at enrollment, we did not exclude women with blood glucose $>100 \mathrm{mg} / \mathrm{dL}$ treated with metformin. However, only a small percentage $(\sim 2 \%)$ of women in this category have received treatment with metformin in recent years, and this would not pertain to women enrolled in the WHI in the 1990s. Finally, the WHI is not a representative sample and, therefore, our results are not generalizable to all postmenopausal women.

In conclusion, in this study, regardless of metabolic health, obesity was associated with increased risk of breast cancer. However, being obese and metabolically unhealthy is associated with the highest risk. Our analysis suggests that, in addition to adiposity, high levels of HOMA-IR may be an independent risk factor for breast cancer. Insulin sensitivity showed a clearer association with risk than the MetS or its individual components. 


\section{Acknowledgments}

Grant Support

This study was supported by institutional funds from the Albert Einstein College of Medicine.

\section{References}

1. Flegal KM, Carroll MD, Kit BK, Ogden CL. Prevalence of obesity and trends in the distribution of body mass index among US adults 199-2010. JAMA 2012;307:491-7. [PubMed: 22253363]

2. Alexander CM, Landsman PB, Teutsch SM, Haffner SM. NCEP defined metabolic syndrome, diabetes, and prevalence of coronary heart disease among NHANES III participants age 50 years and older. Diabetes 2003;52:1210-4. [PubMed: 12716754]

3. Ford ES, Giles WH, Dietz WH. Prevalence of the metabolic syndrome among US adults: findings from the Third National Health and Nutrition Examination Survey. JAMA 2002;287:356-9. [PubMed: 11790215]

4. Grundy SM. The metabolic syndrome: connecting and reconciling cardiovascular and diabetes worlds. J Am Coll Cardiol 2006;47:1093-100. [PubMed: 16545636]

5. Kassi E, Pervanidou P, Kaltsas G, Chrousos G. Metabolic syndrome: definitions and controversies. BMC Med 2011;9:48. [PubMed: 21542944]

6. Munoz-Garach A, Cornejo-Pareja I, Tinahones FJ. Does metabolically healthy obesity exist? Nutrients 2016;8:320.

7. Thomsen M, Nordestgaard BG. Myocardial infarction and ischemic heart disease in overweight and obesity with and without the metabolic syndrome. JAMA Int Med 2014;174:15-22.

8. Kramer CK, Zinman B, Retnakaran R. Aremetabolically healthy overweight and obesity benign conditions? A systematic review and meta-analysis. Ann Intern Med 2013;159:758-69. [PubMed: 24297192]

9. Kip KE, Marroquin OC, Kelley DE, Johnson D, Kelsey SF, Shaw LJ, et al. Clinical importance of obesity versus the metabolic syndrome in cardiovascular risk in women: a report from the Women's Ischemia Syndrome Evaluation (WISE) study. Ciculation 2004;109:706-13.

10. Meigs JB, Wilson PWF, Fox CS, Vasan RS, Nathan DM, Sullivan LM, et al. Body mass index, metabolic syndrome, and risk of type 2 diabetes or cardiovascular disease. J Clin Endocrinol Metab 2006;91:2906-12. [PubMed: 16735483]

11. World Cancer Research Fund/American Institute for Cancer Research. Food, Nutrition, Physical Activity, and the Prevention of Cancer: a Global Perspective. Washington, DC: AICR; 2007.

12. Esposito K, Chiodini P, Capuano A, Bellastella G, Rafaniello C, Giugliano D. Metabolic syndrome and postmenopausal breast cancer: a systematic review and meta-analysis. Menopause 2013;20:1301-9. [PubMed: 23571527]

13. Larsson SC, Mantzoros CS, Wolk A. Diabetes mellitus and risk of breast cancer: a meta-analysis. Int J Cancer 2007;121:856-62. [PubMed: 17397032]

14. Xue F, Michels KB. Diabetes, metabolic syndrome, and breast cancer: a review of the current evidence. Am J Clin Nutr 2007;86:s823-35. [PubMed: 18265476]

15. De Bruijn KMJ, Arends LR, Hansen BE, Leeflang S, Ruiter R, van Eijck CHJ. Systematic review and meta-analysis of the association between diabetes mellitus and incidence and mortality in breast and colorectal cancer. Br J Surg 2013;100:1421-9. [PubMed: 24037561]

16. Moore LL, Chadid S, Singer MR, Kreger BE, Denis GV. Metabolic health reduces risk of obesityrelated cancers in Framingham study adults. Epidemiol Biomarkers Prev 2014;23:2057-65.

17. Gunter MJ, Xie X, Xue X, Kabat GC, Rohan TE, Wassertheil-Smoller S, et al. Breast cancer risk in metabolically healthy but overweight postmenopausal women. Cancer Res 2015;75:270-4. [PubMed: 25593034]

18. Park YM, White AJ, Nichols HB, O'Brien KM, Weinberg CR, Sandler DP. The association between metabolic health, obesity phenotype and the risk of breast cancer. Int J Cancer 2017;140:2657-66. [PubMed: 28268252] 
19. Anderson GL, Manson J, Wallace R, Lund B, Hall D, Davis S, et al. Implementation of the Women's Health Initiative study design. Ann Epidemiol 2003;13:S5-17. [PubMed: 14575938]

20. Langer RD, White E, Lewis CE, Kotchen JM, Hendrix SL, Trevisan M. The Women's Health Initiative Observational Study: baseline characteristics of participants and reliability of baseline measures. Ann Epidemiol 2003;13: S107-21. [PubMed: 14575943]

21. McTiernan A, Kooperberg C, White E, Wilcox S, Coates R, Adams-Campbell LL, et al. Recreational physical activity and the risk of breast cancer in postmenopausal women: the Women's Health Initiative Cohort Study. JAMA 2003;290:1331-6. [PubMed: 12966124]

22. Curb JD, McTiernan A, Heckbert SR, Kooperberg C, Stanford J, Nevitt M, et al. Outcomes ascertainment and adjudiciation methods in the Women's Health Initiative. Ann Epidemiol 13:S122-8. [PubMed: 14575944]

23. Matthews DR, Hosker JP, Rudenski AS, Naylor BA, Treacher DF, Turner RC. Homeostasis model assessment: insulin resistance and $\beta$-cell function from fasting plasma glucose and insulin concentrations in man. Diabetologia 1985;28:412-9. [PubMed: 3899825]

24. Kabat GC, Kim MY, Lane DS, Zaslavsky O, Ho GYF, Luo J, et al. Serum glucose and insulin and risk of cancers of the breast, endometrium, and ovary in postmenopausal women (in press). Europ J Cancer Prev.

25. Renehan AG, Zwalen M, Egger M. Adiposity and cancer risk: new mechanistic insight from epidemiology. Nature 2015;15:484-98.

26. Ish-Shalom D, Christoffersen CT, Vorwerk P, Sacerdoti-Sierra N, Shymko RM, Naor D, et al. Mitogenic properties of insulin and insulin analogues mediated by the insulin receptor. Diabetologia 1997;40 (Suppl 2):S25-31. [PubMed: 9248698]

27. Milazzo G, Giorgino F, Damante F, Sung C, Stampfer MR, Vigneri R, et al. Insulin receptor expression and function in human breast cancer cell lines. Cancer Res 1992;52:3924-30. [PubMed: 1617668]

28. Mawson A, Lai A, Carroll JS, Sergio CM, Mitchell CJ, Sarcevic B. Estrogen and insulin/IGF-1 cooperatively stimulate cell cycle progression in MCF-7 breast cancer cells through differential regulation of c-Myc and cyclin D1. Mol Cell Endocrinol 2005;229: 161-73. [PubMed: 15607540]

29. Simone V,D’ Avenia M, Argentiero A, Felici C, Rizzo RM, De Pergola G, et al. Obesity and breast cancer: molecular interconnections and potential clinical applications. Oncologist 2016;21:404-17. [PubMed: 26865587]

30. Dey N, De P, Leyland-Jones B. PI3-AKT-mTOR inhibitors in breast cancers: from tumor cell signaling to clinical trials. Pharmcol Ther 2017;175:91-106.

31. Chen TYD, Shankar J, Zirpoli G, Roberts MR, Hong CC, Bandera EV, et al. Genetic variants in the mTOR pathway and interaction with body size and weight gain on breast cancer risk in AfricanAmerican and European American women. Cancer Causes Control 2016; 27:965-76. [PubMed: 27314662] 


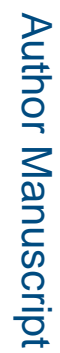

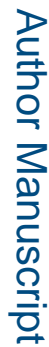

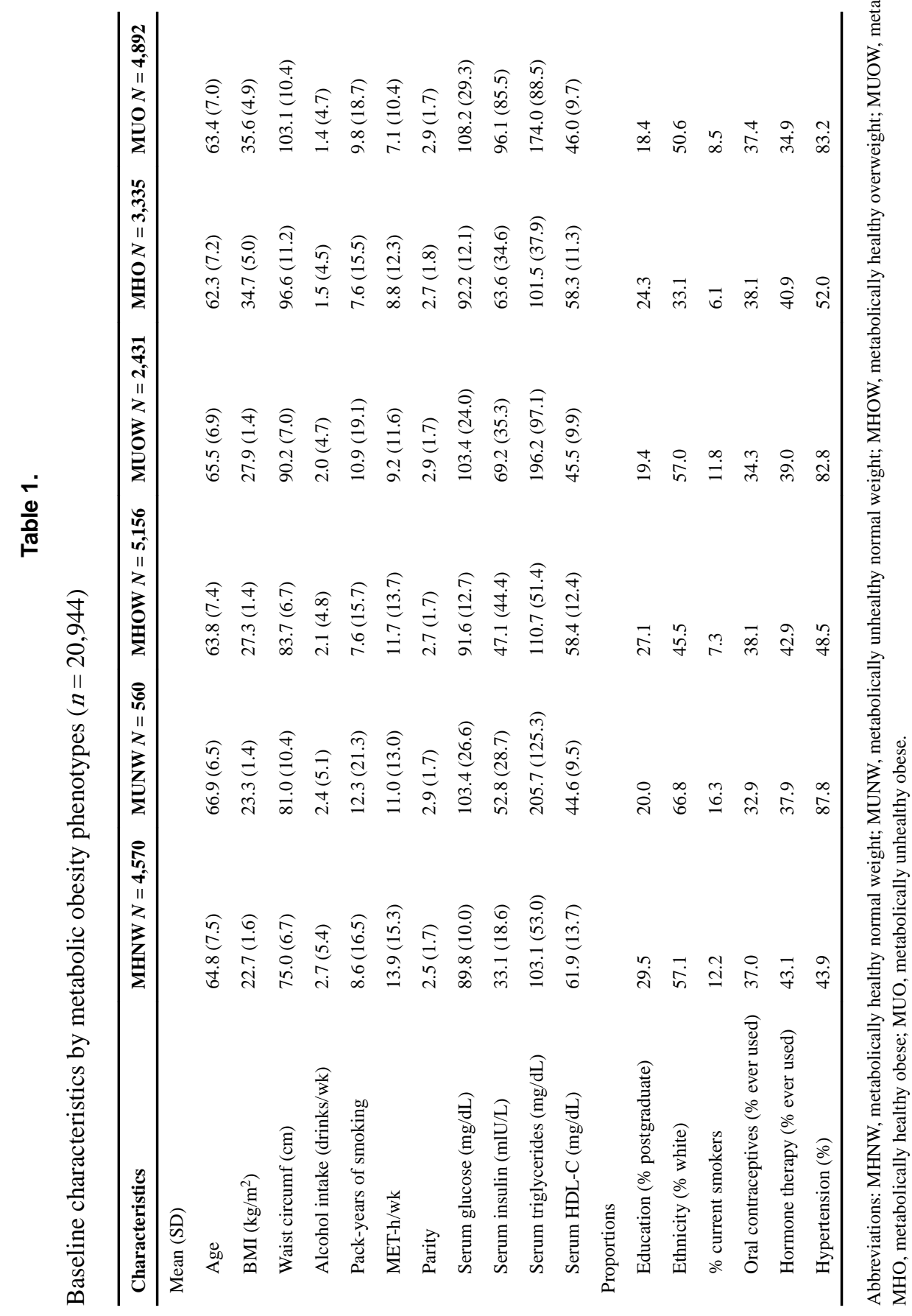

Cancer Epidemiol Biomarkers Prev. Author manuscript; available in PMC 2020 January 28. 
Table 2.

Separate and mutually adjusted associations of BMI and presence of the MetS with breast cancer, Women's Health Initiative CVD Biomarkers Sub-Cohort

\begin{tabular}{|c|c|c|c|}
\hline \multicolumn{4}{|l|}{ A. Separate } \\
\hline BMI $\left(\mathrm{kg} / \mathrm{m}^{2}\right)$ & Cases $(n=1,176)$ & Noncases $(n=19,768)$ & $\mathrm{HR}^{a}(95 \% \mathrm{CI})$ \\
\hline $18.5-<25.0$ & 240 & 4,890 & 1.00 (Ref.) \\
\hline $25.0-<30.0$ & 389 & 7,198 & $1.12(0.95-1.33)$ \\
\hline 230.0 & 547 & 7,680 & $1.51(1.28-1.78)$ \\
\hline$P_{\text {trend }}$ & & & $<0.0001$ \\
\hline \multicolumn{4}{|l|}{ MetS } \\
\hline No & 682 & 12,379 & 1.00 (Ref.) \\
\hline Yes & 494 & 7,389 & $1.28(1.13-1.44)$ \\
\hline \multicolumn{4}{|c|}{ B. Mutually adjusted } \\
\hline BMI $\left(\mathrm{kg} / \mathrm{m}^{2}\right)$ & Cases $(n)$ & Noncases $(n)$ & $\mathrm{HR}^{a}(95 \% \mathrm{CI})$ \\
\hline $18.5-<25.0$ & 240 & 4,890 & 1.00 (Ref.) \\
\hline $25.0-<30.0$ & 389 & 7,1981 & $1.09(0.92-1.30)$ \\
\hline$\geq 30.0$ & 547 & 7,680 & $1.42(1.18-1.70)$ \\
\hline$P_{\text {trend }}$ & & & $<0.0001$ \\
\hline \multicolumn{4}{|l|}{ MetS } \\
\hline No & 682 & 12,379 & 1.00 (Ref.) \\
\hline Yes & 494 & 7,389 & $1.18(1.04-1.34)$ \\
\hline
\end{tabular}

${ }^{a}$ Adjusted for age (continuous), smoking status (never, former, current), pack-years of smoking (continuous), alcohol intake (drinks/week continuous), physical activity (MET-h/wk), age at first birth $(<20,20-29, \geq 30$, missing), age at menarche $(<12,12,13, \geq 14)$, age at menopause ( $<45,45-54, \geq 55$, missing), oral contraceptives (never, ever), hormone therapy (never, ever), parous/nulliparous, family history of breast cancer in first-degree relative (no, yes), history of breast biopsy (no, yes), breastfed for more than 6 months (no, yes), education (less than high school grad, high school grad/some college, college grad, post-college), ethnicity (white, black, other), allocation to the OS or specific arm of clinical trials. 
Table 3.

Metabolic obesity phenotypes defined by presence of the MetS and BMI in relation to risk of breast cancer, in the Women's Health Initiative CVD Biomarkers Sub-Cohort

\begin{tabular}{|c|c|c|c|}
\hline Metabolic phenotypes & Cases, $N$ & Noncases, $N$ & $\operatorname{HR}^{a}(95 \% \mathrm{CI})$ \\
\hline \multicolumn{4}{|c|}{ Total population ( $n$ cases 1,$176 ; n$ noncases 19,819 ) } \\
\hline MHNW & 219 & 4,366 & 1.00 (Ref.) \\
\hline MUNW & 28 & 647 & $0.86(0.51-1.38)$ \\
\hline MHOW & 261 & 4,904 & $1.08(0.90-1.31)$ \\
\hline MUOW & 128 & 2,307 & $1.17(0.93-1.47)$ \\
\hline МHO & 202 & 3,145 & $1.31(1.07-1.61)$ \\
\hline MUO & 345 & 4557 & $1.61(1.34-1.94)$ \\
\hline \multicolumn{4}{|c|}{ Never used hormone therapy ( $n$ cases $714 ; n$ noncases 11,855 ) } \\
\hline MHNW & 121 & 2,486 & 1.00 (Ref.) \\
\hline MUNW & 12 & 337 & $0.76(0.40-1.45)$ \\
\hline MHOW & 148 & 2,800 & $1.14(0.88-1.46)$ \\
\hline MUOW & 75 & 1,411 & $1.18(0.87-1.60)$ \\
\hline MHO & 125 & 1,855 & $1.45(1.11-1.90)$ \\
\hline MUO & 233 & 2,966 & $1.81(1.42-2.29)$ \\
\hline \multicolumn{4}{|c|}{ Never used hormone therapy and not in treatment arms of the HT clinical trials ( $n$ cases $458 ; n$ noncases 7,827 ) } \\
\hline MHNW & 70 & 1,584 & 1.00 (Ref.) \\
\hline MUNW & 9 & 196 & $1.15(0.55-2.40)$ \\
\hline MHOW & 95 & 1,902 & $1.18(0.85-1.63)$ \\
\hline MUOW & 48 & 891 & $1.27(0.86-1.88)$ \\
\hline МHO & 83 & 1,319 & $1.45(1.03-2.04)$ \\
\hline MUO & 153 & 1,935 & $2.01(1.48-2.73)$ \\
\hline \multicolumn{4}{|c|}{ First 3 years of follow-up excluded ( $n$ cases $983 ; n$ noncases 19,410 ) } \\
\hline MHNW & 176 & 4,281 & 1.00 (Ref.) \\
\hline MUNW & 17 & 517 & $0.90(0.54-1.51)$ \\
\hline MHOW & 214 & 4,830 & $1.11(0.90-1.37)$ \\
\hline MUOW & 111 & 2,244 & $1.23(0.96-1.58)$ \\
\hline МHO & 174 & 3,087 & $1.38(1.10-1.73)$ \\
\hline MUO & 291 & 4,451 & $1.65(1.35-2.02)$ \\
\hline
\end{tabular}

Abbreviations: MHNW, metabolically healthy normal weight; MUNW, metabolically unhealthy normal weight; MHOW, metabolically healthy overweight; MUOW, metabolically unhealthy overweight; MHO, metabolically healthy obese; MUO, metabolically unhealthy obese.

${ }^{a}$ Adjusted for age (continuous), smoking status (never, former, current), pack-years of smoking (continuous), alcohol intake (drinks/week continuous), physical activity (MET-h/wk), age at first birth ( $<20,20-29, \geq 30$, missing), age at menarche $(<12,12,13, \geq 14)$, age at menopause $(<45,45-54,255$, missing), oral contraceptives (never, ever), hormone therapy (never, ever), parous/nulliparous, family history of breast cancer in first-degree relative (no, yes), history of breast biopsy (no, yes), breastfed for more than 6 months (no, yes), education (less than high school grad, high school grad/some college, college grad, post-college), ethnicity (white, black, other), allocation to the OS or specific arm of clinical trials. 
Table 4.

Association of combinations of metabolic health defined by HOMA-IR and BMI (normal, overweight, obese) with risk of postmenopausal breast cancer, Women's Health Initiative CVD Biomarkers Sub-Cohort $(n=$ 20,569)

\begin{tabular}{llllll}
\hline $\boldsymbol{(} \boldsymbol{n}$ cases 829; $\boldsymbol{n}$ noncases 13,955) & & & & \\
\hline Low HOMA-IR ${ }^{a} /$ normal weight & 134 & 2,680 & 1.00 & Ref. \\
High HOMA-IR ${ }^{b}$ /normal weight & 91 & 1,919 & 0.95 & $0.72-1.25$ \\
Low HOMA-IR/overweight & 32 & 650 & 0.97 & $0.64-1.46$ \\
High HOMA-IR/overweight & 26 & 650 & 0.85 & $0.55-1.34$ \\
Low HOMA-IR/obese & 152 & 2,796 & 1.17 & $0.91-1.49$ \\
High HOMA-IR/obese & 394 & 5,260 & 1.57 & $1.26-1.95$ \\
\hline
\end{tabular}

NOTE: 41 cases and 385 noncases were missing HOMA-IR. Total $\mathrm{n}$ is reduced because the $2^{\text {nd }}$ quartile was excluded in the analysis. Adjusted for age (continuous), smoking status (never, former, current), pack-years of smoking (continuous), alcohol intake (drinks/week - continuous), physical activity (MET-h/wk), age at first birth ( $<20,20-29, \geq 30$, missing), age at menarche $(<12,12,13, \geq 14)$, age at menopause (<45, 45-54, $\geq 55$,

missing), oral contraceptives (never, ever), hormone therapy (never, ever), parous/nulliparous, family history of breast cancer in first-degree relative (no, yes), history of breast biopsy (no, yes), breastfed for more than 6 months (no, yes), education (less than high school grad, high school grad/ some college, college grad, post-college), ethnicity (white, black, other), allocation to the OS or specific arm of clinical trials.

a Low HOMA-IR defined as lowest quartile.

$b_{\text {High HOMA-IR defined as quartiles } 3 \text { and } 4 .}$ 